\title{
Memória Visuo-Espacial a Curto Prazo: Os Efeitos da Supressão Articulatória e de uma Tarefa Aritmética
}

\author{
Cesar Galera ${ }^{12}$ \\ Universidade de São Paulo, Ribeirão Preto \\ Cláudia Correia Leite Fubs \\ Universidade Paulista, Ribeirão Preto
}

\begin{abstract}
Resumo
Foram realizados dois experimentos para avaliar a natureza da informação armazenada pelo sistema de memória visuo-espacial a curto prazo. No primeiro experimento, uma tarefa de localização espacial foi realizada simultaneamente com tarefas intervenientes de supressão articulatória e de subtração aritmética. A tarefa de supressão articulatória afeta de forma negativa a recordação das letras, mas não a dos padrões visuais. Apesar disso, a recordação das letras se mantém superior à recordação dos padrões visuais. Este resultado sugere que o armazenamento dos padrões visuais não utiliza o laço fonológico, e que a tarefa de supressão articulatória, embora iniba o uso do laço fonológico, pode não inibir o acesso à informação semântica. No segundo experimento estabelecemos o efeito da similaridade visual sobre a capacidade de recordação da posição espacial. Os resultados confirmam o uso de códigos visuais e sugerem que a capacidade de armazenamento de estímulos visuais é limitada, mas não se restringe ao efeito de recência.

Palavras-chave: Memória operacional; memória visuo-espacial; supressão articulatória; tarefa aritmética.
\end{abstract}

\section{Short-Term Visuo-Spatial Memory: The Effect of the Articulatory Suppression and an Arithmetic Task}

\begin{abstract}
We investigated the nature of the information stored in visuo-spatial short-term memory in two experiments. In the first experiment, a spatial localization task was performed simultaneously with articulatory suppression and arithmetical subtraction tasks. The articulatory suppression has a negative effect on the performance with letters, but not with visual patterns. Despite that, the recall of the letters is better than the recall of the visual patterns. This result suggests that the storage of the visual patterns does not use the phonological loop, and that the articulatory suppression, even if it inhibits the use of the phonological loop, cannot inhibit the access to the semantic information. In the second experiment we established the effect of the visual similarity on the capacity of memory for the spatial position. The results confirm the use of visual codes and suggest that the storage capacity of the visual stimuli is limited, but not restricted to the recency items.

Keywords: Working memory; visuo-spatial memory; articulatory suppression; arithmetic task.
\end{abstract}

O enfoque dado por Baddeley (1986, 2000; Baddeley \& Hitch, 1974) ao estudo da memória a curto prazo está centrado no fracionamento deste sistema de memória em subsistemas básicos, especializados no armazenamento e no processamento de diferentes tipos de informação. Atualmente o modelo resultante desse enfoque, o modelo de memória operacional (working memory), envolve quatro subsistemas funcionais. A informação verbal e auditiva é armazenada por um laço fonológico. A memória visuoespacial tem a função de manter e manipular a informação referente aos objetos e às relações espaciais entre eles. O armazenador episódico tem a função de armazenar temporariamente a informação das várias modalidades; as informações provenientes dos outros subsistemas e da

\footnotetext{
${ }^{1}$ Agradecemos a contribuição de Ederaldo J. Lopes e de Renata F. F.Lopes na primeira versão deste trabalho. CNPq (52.2624/95-2).

${ }^{2}$ Endereço para correspondência: Cesar Galera, DPE - FFCLRP-USP, Av. Bandeirantes 3900, 14040 901, Ribeirão Preto, SP. Fone: (16) 602 3760, Fax: (16)6335015.E-mail: algalera@usp.br
}

memória a longo prazo são aglutinadas numa representação episódica unitária. O fluxo de informação vinda do ambiente e da memória a longo prazo está sob a supervisão de um sistema executivo central. Juntos esses subsistemas estariam envolvidos em atividades cognitivas superiores tais como o processamento da linguagem, leitura, solução de problemas e na produção da própria consciência.

O sistema de memória visuo-espacial a curto prazo foi proposto para explicar resultados experimentais que não são facilmente explicáveis em termos da memória verbal. Por exemplo, o desempenho na versão visuo-espacial da tarefa de Brooks (1967) é fortemente perturbado pela execução simultânea de uma tarefa de rastreamento visuoespacial, mas a tarefa de rastreamento não interfere no desempenho da versão verbal da mesma tarefa (Baddeley, Grant, Wight \& Thompson, 1975). Ou seja, parece haver uma sobreposição entre os recursos cognitivos necessários para manter a informação visuo-espacial e os recursos necessários para realizar a tarefa de rastreamento (mas não entre os recursos necessários ao rastreamento e à recordação 
do material verbal). De maneira mais específica, o desempenho na versão visuo-espacial da tarefa de Brooks é fortemente afetado pela realização de uma tarefa de rastreamento acústico, mas não por uma tarefa de vigilância visual, sugerindo que a tarefa de Brooks e a tarefa de rastreamento utilizam os recursos de um sistema de processamento espacial, não visual e não dependente do sistema fonológico (Baddeley \& Liberman, 1980, citados em Baddeley, 1986).

Logie (1986) mostrou que o padrão de interferência obtido quando a tarefa de Brooks é realizada simultaneamente com outras tarefas é determinado em grande parte pela estratégia mnemônica utilizada pelo participante. Seus resultados mostram que a apresentação de estímulos irrelevantes visuais interfere fortemente no desempenho quando o participante utiliza uma estratégia visual, e nesse caso o desempenho não é afetado pela apresentação de estímulos irrelevantes verbais. Por outro lado, quando a estratégia de memorização é verbal o desempenho é fortemente prejudicado pela apresentação de estímulos irrelevantes verbais, mas não pela apresentação de estímulos irrelevantes visuais. Estes resultados sugerem, de acordo com o autor, que o sistema visuo-espacial poderia ser melhor compreendido se fosse subdividido em dois componentes, um dinâmico, voltado ao processamento da informação espacial, e outro passivo, dedicado à informação visual. Tais subsistemas, além de estarem mais ajustados aos resultados experimentais (Logie, 1995), também seriam consistentes com as evidências neuro-fisiológicas de sistemas separados dedicados ao processamento do "que" é, e de "onde" está o estímulo (Milner \& Goodale, 1995; Ungerleider \& Mishkin, 1982).

Duas questões têm recebido atenção nos estudos da memória visual a curto prazo, uma diz respeito à natureza das representações utilizadas, e a outra diz respeito à origem dos recursos necessários à manutenção da informação memorizada. Qual a natureza das representações utilizadas pelo sistema de memória visuo-espacial a curto prazo? $\mathrm{O}$ efeito deletério da similaridade visual sobre o desempenho em tarefas de reconhecimento e de recordação tem sido considerado uma evidência de que a representação dos estímulos é realizada em termos de suas características visuais. $\mathrm{Na}$ tarefa de localização espacial, a recordação de letras e de padrões visuais sem nome é prejudicada quando os estímulos são mais similares entre si (Walker, Hitch \& Duroe, 1993). A taxa de busca na tarefa de reconhecimento de letras também é maior quando o estímulo teste é mais similar aos estímulos memorizados (Galera \& Munhemeze, 1998; Taylor, 1976). Hitch, Halliday, Schaafstal e Schraagen (1988) também mostraram que o desempenho de crianças na tarefa de reconhecimento de figuras também é fortemente afetado por erros de confusão entre figuras visualmente mais similares entre si. De maneira geral, embora os efeitos da similaridade sejam bastante pequenos, estes resultados corroboram a idéia de um sistema de memória a curto prazo baseado em códigos visuais.

Qual a origem dos recursos utilizados pelo sistema de memória visual a curto prazo? $\mathrm{O}$ armazenamento de padrões visuais sem nome pode exigir recursos mais gerais do sistema de memória operacional. Por exemplo, Phillips e Christie (1977a, 1977b) mostraram que a recordação de padrões visuais sem nome é fortemente afetada pela introdução de uma tarefa aritmética que, pelo menos teoricamente, não deveria utilizar recursos do sistema visual. Estes autores determinaram a capacidade da memória visual a curto prazo para listas de estímulos produzidos pelo preenchimento aleatório de oito caselas numa matriz $4 \times 4$. Os resultados mostram que apenas o último estímulo da lista era reconhecido em quase $100 \%$ das provas, enquanto a porcentagem de reconhecimento dos outros estímulos girava em torno de $60 \%$. A introdução de uma tarefa aritmética, a soma de cinco dígitos realizada durante o intervalo de retenção, ou a subtração a partir de um número, de trás para frente, de três em três, eliminou o completamente o efeito de recência, isto é, a vantagem no reconhecimento do último estímulo da lista, mas não afetou o reconhecimento dos primeiros (Phillips \& Christie, 1977b). Parece evidente que os participantes conseguiam manter na mente apenas o último estímulo, mas não podiam fazer o mesmo com os outros (Phillips, 1983). O efeito seletivo da tarefa aritmética sugere, de acordo com os autores, que recursos atencionais externos ao sistema de memória visual são necessários à manutenção do último estímulo da lista. Quando esses recursos são drenados pela tarefa aritmética o sistema não é mais capaz de manter o último estímulo ativo.

As conclusões dos estudos de Phillips e Christie (1977a, 1977b), de que a memória visual a curto prazo tem capacidade para manter apenas o último estímulo da lista, e que este armazenamento exige recursos mais gerais do que aqueles disponíveis ao sistema de memória visual a curto prazo, foram contestadas por D. E. Broadbent e M. H. P. Broadbent (1981), em um estudo que também utilizou uma tarefa de reconhecimento com padrões visuais sem nome. Os resultados mostraram um efeito de recência ampliado: os participantes eram mais precisos quando o teste estava entre os últimos estímulos da lista. Alem disso, a introdução de uma tarefa de contar de 1 a 6 , em voz alta, durante a apresentação dos estímulos, ou a tarefa de contar o número de símbolos num display durante o intervalo de retenção, afetou fortemente o desempenho geral na tarefa, mas deixou intacto o efeito de recência, isto é, a taxa de 
recordação dos últimos estímulos continuou maior do que a taxa de recordação dos primeiros. Isso indica que os mesmos recursos seriam utilizados pela tarefa de contar e pela manutenção dos itens memorizados em geral, incluindo os estímulos de recência.

Um estudo recente realizado por Lee e Kang (2002) também permite supor que a tarefa aritmética de subtração utiliza recursos da memória visuo-espacial. Estes autores investigaram a relação entre tarefas aritméticas de subtração e de multiplicação e os sistemas de memória fonológica e visuo-espacial utilizando o paradigma de tarefa dupla. A introdução de uma tarefa de supressão verbal teve um efeito deletério sobre a tarefa de multiplicação, mas não afetou a tarefa de subtração. Por outro lado, manter na memória a posição na qual um padrão visual fora apresentado prejudicou a realização da tarefa de subtração, mas não teve efeito significante sobre o desempenho na tarefa de multiplicação. Estes resultados indicam que a tarefa de subtração utiliza recursos relacionados ao sistema visuo-espacial.

Em resumo, existem fortes evidências de um sistema de armazenamento visuo-espacial a curto prazo. Existem também evidências de que este sistema pode ter componentes específicos para o processamento de características visuais e espaciais. Mas existem ainda dúvidas quanto à natureza dos recursos empregados por esses componentes. Neste estudo investigamos a natureza dos recursos empregados pelo sistema de memória visuo-espacial a curto prazo, determinando o efeito de duas tarefas de supressão, uma verbal e uma aritmética, sobre reconhecimento de letras e de padrões visuais numa tarefa de localização espacial.

\section{Experimento 1}

Neste experimento, procuramos avaliar a natureza dos recursos disponíveis ao sistema de memória visual a curto prazo utilizando uma tarefa de supressão articulatória e uma tarefa aritmética de subtração. Vários estudos têm mostrado que a tarefa de supressão articulatória inibe a re-codificação fonológica de estímulos apresentados de forma visual. Por exemplo, os efeitos do comprimento da palavra, ou da similaridade fonológica, que dependem do laço fonológico, desaparecem quando os participantes repetem sílabas ou palavras sem sentido durante a apresentação dos estímulos (Baddeley, 1986). Uma vez que a supressão articulatória impede a recitação do nome do estímulo, nossa suposição era que a tarefa de supressão verbal afetaria o reconhecimento das letras, mas não o dos padrões visuais sem nome. Alem disso, supúnhamos que, se as letras são codificadas tanto em termos verbais como em termos visuais, a tarefa de supressão verbal, ao eliminar o acesso desses estímulos ao laço fonológico, deveria igualar as taxas de reconhecimento de letras e de padrões visuais.

A tarefa aritmética de subtração pode utilizar tanto os recursos visuo-espaciais (Lee \& Kang, 2002) necessários ao armazenamento do local em que um estímulo foi apresentado, como pode utilizar os recursos necessários ao armazenamento de padrões visuais estáticos, como pode ter acontecido nos estudos de Philips e Christie (1977b) e de por D. E. Broadbent e M. H. P. Broadbent (1981). Dessa forma, a introdução de uma tarefa de subtração deve afetar apenas a recordação dos padrões visuais sem nome, no caso de ocupar os recursos necessários a um componente visual do sistema, como pode afetar tanto a recordação de letras como dos padrões visuais, caso partilhe recursos com o componente espacial do sistema de memória visuoespacial.

\section{Método}

\section{Participantes}

Doze estudantes, oito alunos do primeiro semestre de Psicologia da Universidade Paulista - Ribeirão Preto, e quatro alunos de uma escola particular de Ensino Médio de Ribeirão Preto, com idade variando de 16 a 22 anos, todos com visão normal ou corrigida, foram voluntários em duas sessões experimentais.

\section{Planejamento}

Os participantes passaram por duas condições experimentais, uma com letras e outra com padrões visuais como estímulos. Nestas duas condições foram manipulados os seguintes fatores experimentais: posição serial do estímulo teste na seqüência apresentada (primeiro, segundo, terceiro, quarto estímulo), posição espacial na qual o estímulo teste foi apresentado (esquerda, centro esquerda, centro direita, direita), conjunto apresentado (quatro conjunto possíveis) e similaridade (alta, baixa). Cada um dos 128 tratamentos, resultantes da combinação desses quatro fatores (4 ordens x 4 posições $\mathrm{x} 4$ conjuntos $\mathrm{x} 2$ similaridades), foram apresentados duas vezes, totalizando 256 provas em cada condição experimental. Em cada condição experimental foram selecionadas aleatoriamente 80 provas a serem realizadas com a tarefa aritmética, 80 com a tarefa de supressão articulatória e 96 provas de controle. O tipo de estímulo (letra ou padrão visual) foi manipulado entre sessões experimentais. Os outros tratamentos mudaram aleatoriamente de prova para prova.

\section{Material e Estímulos}

Foram utilizados como estímulos 16 letras (Figura 1a) e 16 padrões visuais, criados pelo preenchimento semi- 
aleatório de oito caselas de uma matriz 4 x 4 (Figura 1b). Padrões visuais pertencentes a uma mesma linha foram gerados de forma que fossem mais semelhantes entre si do que em relação a estímulos pertencentes a linhas diferentes. Dessa forma os estímulos pertencentes à mesma linha tinham mais componentes em comum entre si do que estímulos pertencentes a linhas diferentes, definindo respectivamente conjuntos de estímulos de similaridade alta e baixa. De forma semelhante, as letras pertencentes a uma mesma linha foram selecionadas de forma que tivessem mais características comuns entre si e diferentes das letras de outras linhas, definindo dois níveis de similaridade: alta, entre letras da mesma linha, e baixa, para letras de linhas diferentes. A dimensão dos estímulos nos diferentes subgrupos de letras variou ligeiramente. As letras formadas por linhas retas mediam aproximadamente $18 \mathrm{~mm} \times 21 \mathrm{~mm}$; as letras formadas linhas curvas mediam em torno de $22 \mathrm{~mm}$ x $22 \mathrm{~mm}$.

Os estímulos foram apresentados em preto sobre a tela branca de um monitor SVGA, controlado por um microcomputador modelo IBM-PC. As posições nas quais os estímulos foram apresentados eram marcadas por quatro quadrados, com $4,5 \mathrm{~cm}$ de lado, definidos apenas pelo contorno preto, dispostos numa linha horizontal, no centro da tela do monitor. A distância entre dois quadrados vizinhos era de $2 \mathrm{~cm}$. O estímulo teste era apresentado num quadrado adicional, situado no centro da tela, $4 \mathrm{~cm}$ abaixo das posições onde eram apresentados os estímulos. Estes foram observados a uma distância aproximada de $50 \mathrm{~cm}$.

\section{Procedimento}

A tarefa de localização espacial utilizada neste estudo consiste na apresentação seqüencial de quatro estímulos em quatro posições na tela do monitor, seguidos por um estímulo teste, apresentado em uma posição neutra. A tarefa do participante é identificar a posição espacial na qual o estímulo teste foi originalmente apresentado. Cada estímulo da seqüência foi apresentado para memorização durante um intervalo de 1 segundo, com um intervalo de 0,5 segundo entre cada estímulo. Meio segundo após a apresentação do último estímulo da seqüência, o estímulo teste era apresentado na posição neutra e permanecia na tela até que o participante emitisse sua resposta sobre a posição na qual esse estímulo havia sido apresentado. $\mathrm{O}$ participante deu sua resposta no teclado do computador, pressionando as teclas $\mathrm{S}$ (teste na posição à esquerda da tela), $F$ (teste na posição centro esquerda), com os dedos anular e indicador da mão esquerda, e as teclas J (teste na posição centro direita) e L (teste na posição à direita) com os dedos indicador e anular da mão direita, respectivamente. Para iniciar a prova seguinte, o participante deveria pressionar a barra de espaço. Dois segundos depois, tinha início uma nova prova.

Nas provas com a tarefa aritmética, um número de três dígitos era apresentado, no centro da tela, dois $\mathrm{cm}$ acima de onde seriam apresentados os estímulos, dois segundos antes que estes fossem apresentados. Tão logo fosse apresentado esse número, o participante deveria iniciar uma contagem em ordem decrescente, de dois em dois, em voz alta, de forma pausada e regular, enquanto eram apresentados os estímulos da seqüência, até que o estímulo teste fosse apresentado e o participante identificasse a posição na qual este havia sido apresentado. Nas provas com tarefa de supressão verbal, um conjunto de três silabas com três letras (Ex.: bla bla bla) era apresentado no mesmo local, por dois segundos, antes do início da apresentação dos estímulos. O participante deveria repetir o conjunto de sílabas em voz alta, de forma pausada e regular, enquanto eram apresentados os estímulos da seqüência, até que o estímulo teste fosse exibido e o participante desse sua resposta. Nas provas de controle, um asterisco $\left(^{*}\right)$ era apresentado no centro da tela dois segundos antes da apresentação dos estímulos. O experimentador permanecia ao lado do participante durante a sessão experimental.

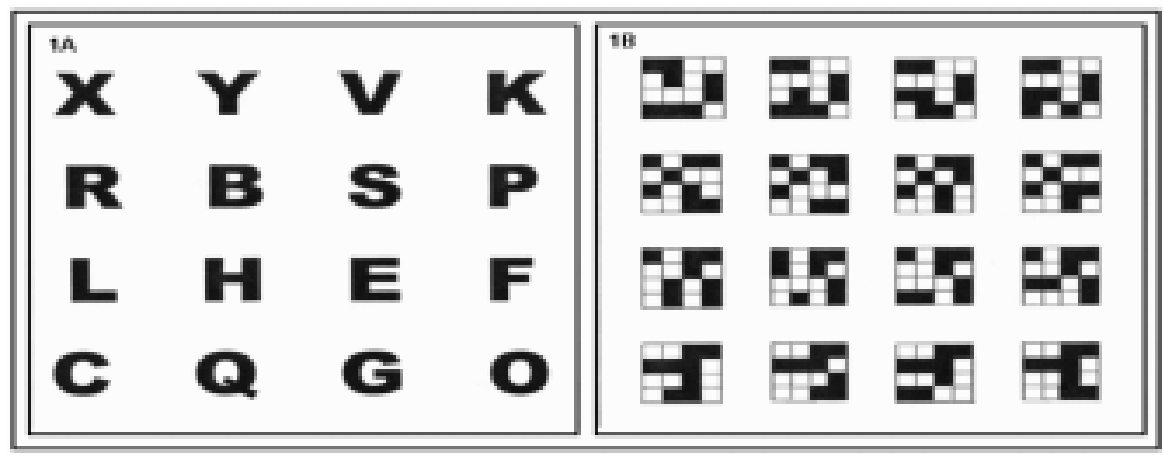

Figura 1. Experimento 1. Letras (1a) e padrões visuais (1b) utilizados como estímulos. Conjuntos formados por estímulos pertencentes à mesma linha foram definidos como de similaridade alta. Conjuntos formados por estímulos pertencentes a uma mesma coluna foram definidos como de similaridade baixa. 


\section{Resultados e Discussão}

As primeiras 10 provas de cada sessão foram consideradas como treino e não foram levadas em conta para a análise dos resultados. Os dados obtidos na condições experimentais com letras e padrões visuais foram analisados separadamente. Em cada condição a porcentagem de respostas corretas foi submetida a uma análise de variância levando-se em conta as tarefas intervenientes realizadas durante a tarefa de localização espacial (supressão verbal, aritmética e controle), a similaridade entre estímulos (alta, baixa), a posição serial na qual o estímulo teste foi apresentado (primeiro, segundo, terceiro ou quarto estímulo) e a posição espacial na qual o estímulo teste foi apresentado (esquerda, centro esquerda, centro direita e direita). As porcentagens de respostas corretas para letras e padrões visuais, em função da posição serial do estímulo teste, nas três tarefas estudas são apresentadas na Figura 2.

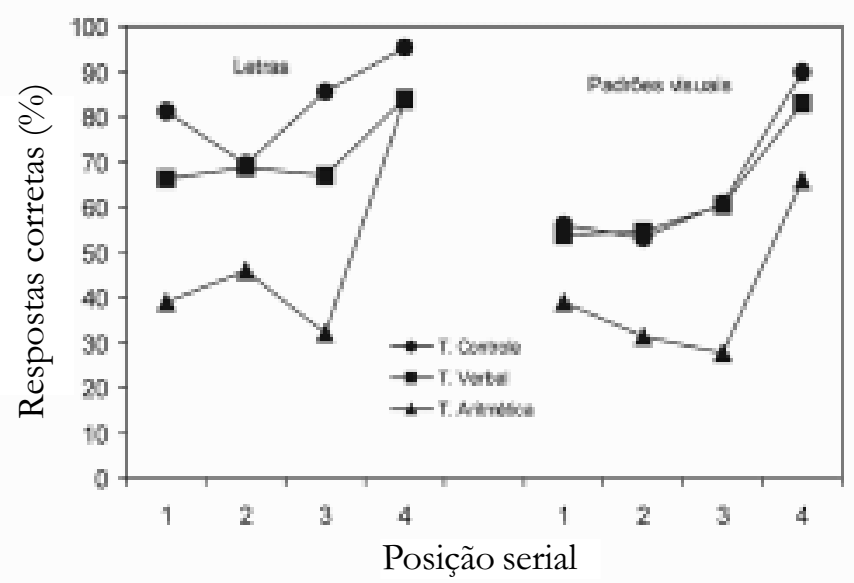

Figura 2. Experimento 1. Porcentagem de respostas corretas obtidas com letras e com padrões visuais sem nome, em função da posição serial do estímulo teste nas seqüências apresentadas.

\section{Letras}

A taxa de recordação correta da posição das letras foi afetada pelas tarefas intervenientes $(F(2,22)=94,26$, $p<0,0001)$. A taxa de acertos é maior $(82 \%)$ nas provas em que o participante pode articular livremente o nome das letras apresentadas. Nas provas em que a tarefa de supressão articulatória foi utilizada a taxa de acertos foi de $71 \%$. Nas provas em que foi realizada a tarefa aritmética a taxa de acertos ficou em 49\%. O desempenho não foi afetado de forma significante pela similaridade entre os estímulos $(F(3,33)<1)$.

O efeito principal da posição serial é significante $(F(3$, $33)=22,78, p<0,001)$, indicando um forte efeito de recência restrito ao último estímulo. Este estímulo é recordado corretamente em $87 \%$ das provas, enquanto que a recordação dos três primeiros fica em torno de $61 \%$. As taxas de recordação dos três primeiros estímulos não diferem entre si de forma significante, tal como indicado pelo teste LSD post-hoc $(p \geq 0,714)$.

As tarefas intervenientes tiveram efeitos diferenciados em função da posição serial do estímulo teste $(F(6,66)=8,68$, $p<0,001)$, prejudicando mais a recordação dos primeiros estímulos apresentados. Na tarefa de controle, a taxa de recordação do último estímulo é maior do que a de todos os outros (LSD, todas as comparações $\operatorname{com} p \leq 0,001)$, e a taxa de recordação do penúltimo é maior do que a do segundo $(p=0,002)$, de forma que o efeito de recência engloba os últimos dois estímulos da seqüência apresentada. Nas provas com a tarefa de supressão articulatória verbal houve, em comparação com as provas de controle, uma diminuição média de 11 pontos percentuais na taxa de acertos (15 para o primeiro; 0 para o segundo; 19 para o terceiro; 11 para o quarto estímulo apresentado). Mesmo assim o efeito de recência confere ao último estímulo uma vantagem de 17 pontos percentuais sobre os três primeiros.

$\mathrm{Na}$ tarefa aritmética, comparada com a tarefa de controle, houve uma redução média de 40 pontos percentuais na taxa de acertos dos três primeiros estímulos apresentados, e uma redução de 12 pontos percentuais na taxa de acertos do último estímulo. Neste caso o efeito de recência dá ao último estímulo uma vantagem de 45 pontos percentuais sobre os estímulos anteriores.

Uma análise das respostas corretas em função da posição espacial do estímulo teste revela que a taxa de respostas corretas é maior quando o alvo é apresentado nas posições mais à esquerda da tela $(F(3,33)=4,2$, $p=0,013)$, sugerindo um possível efeito da "recitação" no sentido da leitura, da esquerda para a direita.

\section{Análise dos Erros}

A análise das respostas incorretas, que levou em conta apenas a distribuição espacial das respostas, também revela que os erros tendem a ser mais concentrados nas posições adjacentes àquela na qual o alvo foi apresentado $(F(2$, $22)=4,01, p=0,030$ ). Nas provas em que o alvo foi apresentado em posições estremas, à esquerda ou à direita, a posição adjacente recebe $80 \%$ das respostas incorretas. Nas provas em que o alvo foi apresentado numa posição central (centro esquerda ou centro direita) a posição adjacente central recebe $50 \%$ das respostas incorretas $(F(2,22)=12,98$, $p<0,001)$.

A análise das respostas incorretas em função da posição serial revela que os participantes são menos propensos a indicar a posição na qual o último estímulo foi apresentado 
como a posição do estímulo teste. A análise dos erros cometidos quando o alvo era o primeiro, segundo ou terceiro estímulo apresentado mostram que a posição na qual o quarto estímulo foi apresentado só é confundida com a posição do alvo em $8 \%$ das provas incorretas $(F(3,33)=22,53, p<0,001)$. Este dado é importante porque o efeito de recência poderia ser o resultado de uma estratégia de "chutar" a posição do último estímulo apresentado quando houvesse incerteza sobre a resposta correta, mas isto não aconteceu em nosso estudo.

Os erros tendem a se agrupar também em função da proximidade temporal com o alvo $(F(6,66)=13,38, p<0,001)$, isto é, nas respostas incorretas o participante tende a escolher a posição do estímulo que se seguiu ou que antecedeu o alvo. Quanto o alvo foi o primeiro estímulo apresentado, $58 \%$ das respostas incorretas apontam a localização do segundo estímulo apresentado como sendo a localização do alvo. Quando o alvo foi o segundo estímulo apresentado, as posições ocupadas pelo primeiro e pelo terceiro estímulos receberam, respectivamente, 44 e $45 \%$ dos erros cometidos. Quando o alvo é o terceiro estímulo apresentado 53\% das respostas incorretas apontam a posição ocupada pelo segundo estímulo como aquela na qual o alvo teria sido apresentado.

\section{Padrões Visuais}

A taxa de recordação da posição dos padrões visuais foi afetada de forma significante pelas tarefas intervenientes $(F(2,22)=58,2, p<0,001)$. As taxas de acertos nas provas de controle $(65 \%)$ e nas provas com supressão articulatória $(63 \%)$ não são diferentes entre si $(p=0,429)$, mas são maiores do que a taxa de acertos de $41 \%$ obtida nas provas com a tarefa aritmética $(p<0,0001)$. A taxa média de acertos em função da posição serial mostra que a posição do último estímulo apresentado foi recordada corretamente em 79\% das provas, enquanto que a recordação dos três primeiros estímulos fica em torno de $48 \%(F(3,33)=20,92, p<0,001)$. A taxa de acertos não foi afetada pela similaridade entre estímulos $(F(1,11)=3,06, p=0,110)$.

Nossa suposição, de que a tarefa de supressão verbal não teria efeito nas provas realizadas com figuras sem nome, se confirmou plenamente. Comparada com a tarefa de controle, a supressão da articulação provocou uma diminuição irrisória de dois pontos percentuais na taxa de acertos. Supondo-se que a articulação seria necessária para a tradução da informação visual para uma forma de código fonológico (Baddeley, 1986), a ausência do efeito de supressão mostra que a codificação fonológica não contribui para o reconhecimento dos padrões visuais sem nome. Este resultado nos permite concluir que as figuras foram mantidas no armazenamento visual a curto prazo, independente do sistema articulatório fonológico.

A tarefa aritmética provocou, em comparação com as provas controle, uma redução de aproximadamente 17 pontos percentuais na taxa de acertos do primeiro estímulo apresentado, 22 pontos percentuais na taxa de acertos do segundo estímulo, e um efeito maior, de 33 pontos percentuais sobre o terceiro estímulo. A redução na taxa de acerto do último estímulo foi de 24 pontos percentuais. Esse efeito diferencial da tarefa aritmética sobre a taxa de reconhecimento em função da posição serial ocupada pelo estímulo teste é confirmado pela interação significativa entre esses fatores $(F(6,66)=2,53, p<0,029)$.

\section{Análise dos Erros}

Uma análise da distribuição das respostas nas diferentes posições espaciais revela também que os erros tendem a ser mais concentrados nas posições adjacentes à posição do alvo $(F(2,22)=8,3, p=0,015)$. Nas provas em que o alvo foi apresentado em posições estremas, à esquerda ou à direita, a posição adjacente recebe $69 \%$ das respostas incorretas. Nas provas em que o alvo foi apresentado numa posição central (centro esquerda ou centro direita) a posição adjacente central recebe $88 \%$ das respostas incorretas $(F(2,22)=10,3$, $p=0,001)$.

Os participantes são menos propensos a indicar a posição na qual o último estímulo foi apresentado como a posição do estímulo teste $(F(3,33)=21,73, p<0,0001)$, uma estratégia que poderia inflar o efeito de recência. Uma proporção significativa dos erros tende a se agrupar também em função da proximidade temporal com o alvo $(F(6,66)=2,60$, $p<0,025)$. Quando o alvo foi o primeiro estímulo apresentado, a posição do segundo estímulo é a escolhida em $42 \%$ das respostas incorretas. Aproximadamente $80 \%$ das repostas incorretas apontam a posição do primeiro e do terceiro estímulos, quando o alvo é o segundo estímulo apresentado. Quando o alvo é o terceiro estímulo da seqüência, a posição na qual o segundo estímulo foi apresentado recebe $38 \%$ das respostas incorretas.

A rigor, numa tarefa com quatro respostas possíveis, a taxa de respostas corretas ao acaso deve girar em torno de $25 \%$. É possível que estratégias mais elaboradas do que responder ao acaso permitam elevar essa proporção. Por exemplo, se o estímulo teste não é o último estímulo apresentado, o participante pode levar em conta essa informação e restringir suas opções apenas aos três estímulos anteriores, elevando sua chance de acertar a resposta ao acaso em 33\% das provas (Walker \& cols., 1993). Nossos resultados mostram que as informações disponíveis ao participante permitiram taxas de acertos superiores ao que seria esperado caso a estratégia acima tivesse sido utilizada. Taxas próximas ao acaso foram obtidas apenas em algumas situações específicas. Com as letras, a 
introdução da tarefa aritmética reduziu a taxa de reconhecimento do terceiro estímulo apresentado para $32 \%$, o que não difere de forma significante da taxa que seria esperada ao acaso $(t(11)=1,65, p=0,060)$. Com padrões visuais, taxas de reconhecimento de $32 \%(t(10)=1,81, p=0,090)$, obtida frente ao segundo estímulo apresentado, e de $26 \%$ $(t(10)=0,28, p=0,390)$, obtida frente ao terceiro estímulo apresentado, também não podem ser consideradas diferentes do que seria esperado ao acaso.

A ausência do efeito da supressão articulatória sobre a recordação dos padrões visuais sem nome confirma que o armazenamento deste tipo de estímulo não utiliza o laço fonológico. Não podemos afirmar, no entanto, que a supressão articulatória nas provas com letras tenha restringido os participantes a utilizarem apenas o rascunho visuo-espacial a curto prazo para armazenar a informação visual das letras. Nossa suposição inicial era que as letras, despidas de suas características fonológicas pela tarefa de supressão, se comportariam apenas como figuras visuais, mas isto não é verdade. A taxa de acertos obtida nas provas com letras é maior, aproximadamente $9 \%$, do que a taxa de acertos obtida com as figuras, tanto para a tarefa de supressão verbal $(p=0,009)$ como na tarefa aritmética $(p=0,007)$. Por que as letras têm uma vantagem em relação aos padrões, tanto na supressão verbal como na tarefa aritmética? Se, de fato as letras foram tratadas da mesma forma que as figuras sem nome, este resultado sugere que o armazenador visual a curto prazo pode ser sensível a estímulos super aprendidos. Também é possível que as letras sejam nomeadas automaticamente; a supressão articulatória pode impedir a recitação, mas pode não impedir que as letras sejam nomeadas, permitindo-lhes acesso ao processamento semântico e a um desempenho superior.

\section{Experimento 2}

O efeito da similaridade é importante para compreender a natureza do sistema de memória visuo-espacial a curto prazo. Vários estudos têm mostrado que a similaridade afeta a recordação de letras e de padrões visuais (Logie, 1995; Walker \& cols., 1993). Este efeito é considerado uma evidência de um sistema de memória a curto prazo baseado em códigos visuais. Nós não obtivemos um efeito significante da similaridade. Isto se deve a uma característica dos estímulos ou do código utilizado pelo sistema de memória? É possível que não tenhamos obtido um efeito significante deste fator porque as diferenças entre os níveis de similaridade utilizados não tenham sido grandes o suficiente. Dada a sua importância, o efeito da similaridade foi reavaliado em um novo experimento utilizando letras e padrões visuais com níveis mais acentuados de similaridade/dissimilaridade.

\section{Método}

\section{Participantes}

Onze estudantes do curso de Psicologia da Universidade Paulista - Ribeirão Preto, com idade variando de 19 a 22 anos, todos com visão normal ou corrigida.

\section{Material e Estímulos}

Foram utilizados como estímulos 16 letras e 16 padrões visuais (Figura 3), iguais àqueles empregados por Walker $\mathrm{e}$ colaboradores (1993). A dimensão dos estímulos nos diferentes subgrupos variou ligeiramente, sendo que o tamanho máximo foi de $6 \mathrm{~mm} \times 20 \mathrm{~mm}$. As letras formadas por linhas retas mediam $10 \mathrm{~mm} \times 15 \mathrm{~mm}$; as letras formadas linhas curvas mediam $15 \mathrm{~mm} \times 15 \mathrm{~mm}$.

Como no experimento anterior, as letras e padrões visuais foram combinadas em quatro subconjuntos de quatro estímulos cada um. Estímulos pertencentes à mesma linha na Figura 3 foram considerados como mais similares entre si do que estímulos pertencentes a linhas diferentes. Desta forma foram definidos conjuntos de estímulos de similaridade alta (pertencentes à mesma linha) e de similaridade baixa (pertencentes à mesma coluna). $\mathrm{O}$ equipamento e os outros detalhes dos estímulos utilizados são os mesmos que no experimento anterior.

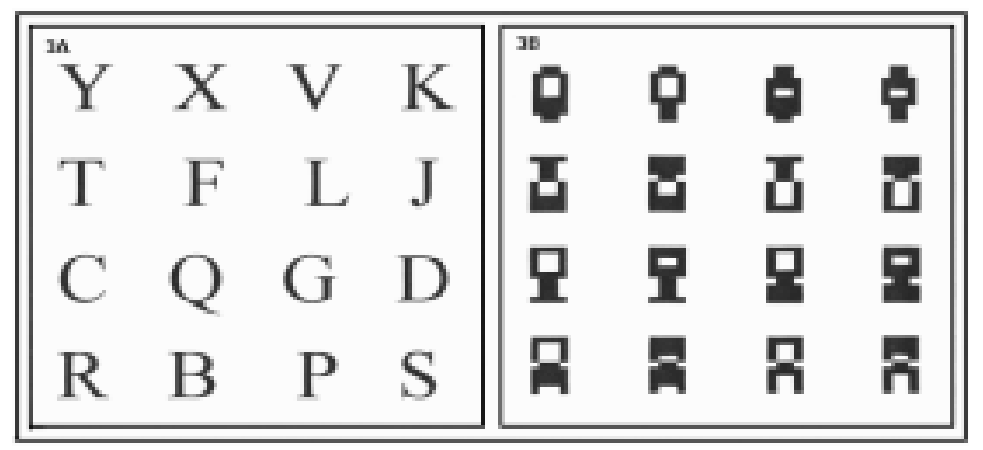

Figura 3. Experimento 2. Letras (3A) e padrões visuais (3B) utilizados como estímulos. Conjuntos formados por estímulos pertencentes à mesma linha foram definidos como de similaridade alta. Conjuntos formados por estímulos pertencentes a uma mesma coluna foram definidos como de similaridade baixa (estímulos baseados em Walker \& cols., 1993). 


\section{Planejamento/Procedimento}

A tarefa de localização espacial também foi utilizada neste experimento. Os participantes passaram por duas condições experimentais, uma tendo letras e outra tendo padrões visuais sem nome como estímulos. Nessas condições foram manipulados os seguintes fatores experimentais: posição serial do estímulo teste na seqüência de estímulos (primeiro, segundo, terceiro ou quarto), posição do estímulo teste na tela (esquerda, centro esquerda, centro direita e direita) e similaridade entre os estímulos da seqüência (alta, baixa). Em cada uma das condições experimentais cada participante realizou 256 provas, resultantes da aplicação repetida, duas vezes, de todos os tratamentos experimentais ( 4 ordens $\mathrm{x} 4$ posições $\mathrm{x} 2$ níveis de similaridade $\mathrm{x} 4$ conjuntos). Outros detalhes do procedimento foram iguais aos do experimento anterior.

\section{Resultados e Discussão}

As primeiras 10 provas de cada condição foram consideradas como treino e não foram levadas em conta nas análises realizadas. Os resultados obtidos nas duas condições experimentais (Figura 4) foram submetidos, separadamente, a uma análise de variância com medidas repetidas nos fatores posição serial do estímulo teste, similaridade visual, e posição espacial na qual o estímulo teste foi apresentado.

\section{Letras}

A recordação das duas últimas letras apresentadas em cada serie é melhor do que a das duas primeiras $(F(3,30)=12,93, p<0,001)$, revelando um efeito de recência com dois itens. A taxa de recordação de letras com similaridade alta ( $81 \%$ ) é menor do que a taxa de recordação das letras com similaridade baixa $(84 \%) \quad(F(1,10)=17,39$, $p=0,002)$. Essa diferença se restringe aos dois últimos estímulos apresentados, pois penas os estímulos da recência foram afetados pela similaridade $(F(3,30)=4,27, p=0,013)$. Para estes estímulos a taxa de recordação é $7 \%$ menor quando a similaridade é alta $(p \leq 0,009)$. Este efeito da similaridade sobre os últimos estímulos interfere sobre o efeito de recência: quando a similaridade é baixa o efeito de recência engloba os dois últimos estímulos, mas quando a similaridade é alta o efeito de recência se restringe apenas ao último estímulo.

Embora a posição espacial na qual o estímulo teste foi apresentado não tenha tido um efeito principal significante $(p=0,34)$, este fator interage de forma significante com a similaridade $(F(3,30)=5,80, p<0,001)$. A taxa de recordação

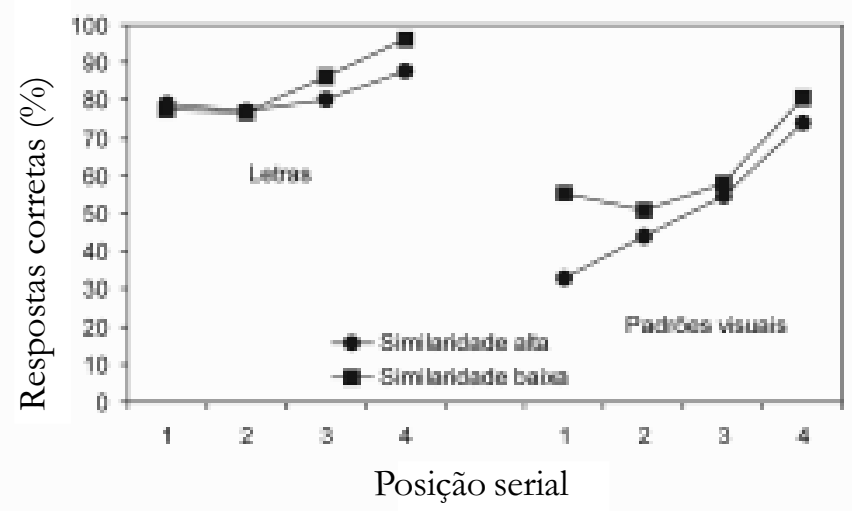

Figura 4. Experimento 2. Porcentagem de respostas corretas obtidas com letras e com padrões visuais sem nome, em função da posição serial do estímulo teste nas seqüências apresentadas.

de um estímulo de similaridade baixa é pior na posição centro esquerda (82\%) e melhor na posição à direita (88\%). Já os estímulos de similaridade alta tem a melhor taxa de reconhecimento na posição centro esquerda $(86 \%)$ e as piores nas posições à esquerda $(75 \%)$ e à direita $(81 \%)$.

\section{Análise de Erros}

A taxa de respostas incorretas varia de maneira sistemática em função da posição espacial ocupada pelo estímulo teste. Quando o teste é apresentado numa das posições extremas da configuração (esquerda ou direita), a posição adjacente é mais escolhida $(46 \%)$ do que as outras posições quando o participante dá uma resposta incorreta $(F(2,20)=14,6$, $p=0,001)$. Quando o alvo é apresentado numa posição central , 48\% dos erros são cometidos na posição adjacente central $(F(2,20)=4,2, p=0,030)$. A proporção de erros em torno da posição na qual o teste é apresentado sugere que essas respostas não foram completamente ao acaso.

A taxa de resposta dos itens apresentados em primeiro lugar, em torno de 78\%, mostra que o desempenho está acima do que seria esperado se os participantes respondessem ao acaso, sugerindo que informações referentes aos primeiros estímulos apresentados permanecem úteis por um período de aproximadamente seis segundos, compreendido entre a apresentação do primeiro estímulo e a apresentação do estímulo teste.

\section{Padrões Visuais}

A análise realizada com os padrões visuais sem nome confirma que a taxa de acertos também é maior para os dois últimos estímulos apresentados $(F(3,30)=23,59$, $p<0,0001)$. Novamente, a porcentagem de recordações corretas é maior quando os estímulos são pouco similares entre si $(64,7 \%)$ do que quando são mais similares $(56,2 \%)$ $(F(1,10)=9,07, p=0,013)$. O efeito da similaridade varia em 
função da ordem de apresentação dos estímulos $(F(3,30)=4,43, p=0,011)$, mas, ao contrário do que acontece com as letras, o efeito da similaridade é maior sobre o primeiro estímulo a ser apresentado. A taxa de recordação de um estímulo de similaridade alta apresentado em primeiro lugar é $22 \%$ menor do que se o estímulo for de similaridade baixa $(\phi<0,001)$. A importância da similaridade visual diminui para os últimos estímulos apresentados. Embora o aumento da similaridade provoque uma queda média de $6 \%$ nas respostas corretas para os três últimos estímulos apresentados, essa diferença não pode ser considerada significante (todos com $p>0,1)$. Este efeito maior da similaridade nos itens pré recência não parece ser um resultado fortuito, uma vez que também é encontrado no estudo de Walker e colaboradores (1993).

O número de estímulos abrangidos pelo efeito de recência depende da similaridade entre os estímulos apresentados. Da mesma forma que nas provas com letras, o efeito de recência se restringe ao último estímulo apresentado na seqüência de similaridade baixa. A taxa de recordação deste estímulo é melhor do que a de todos os estímulos anteriores (todas as comparações $\operatorname{com} p<0,001$ ), e não existe diferença significante entre as taxas de recordação dos três primeiros estímulos. Nas provas com estímulos de similaridade alta, a taxa de recordação do último estímulo (82\%) é maior do que a do penúltimo $(60 \%, p<0,001)$; a taxa de recordação do penúltimo é maior do que a do segundo $(45 \%, p<0,001)$, e a taxa de recordação deste é maior do que a do primeiro estímulo apresentado $(35 \%$, $p<0,001)$.

A taxa de respostas corretas também é afetada pela posição espacial na qual o estímulo teste foi apresentado $(F(3,30)=2,97, p=0,047)$. A taxa de acertos é melhor na posição centro esquerda $(60 \%)$ do que na posição à direita $(52 \%)$, mas não é significante a diferença entre as taxas de acertos na posição esquerda $(56 \%)$ e centro direita $(57 \%)$.

\section{Análise de Erros}

A taxa de respostas incorretas também está associada à posição espacial na qual o alvo é apresentado. Quando o alvo é apresentado em uma das posições extremas da configuração (esquerda ou direita), a posição adjacente recebe $37 \%$ dos erros $(F(2,20)=13,1, p=0,001)$. Quando o alvo é apresentado em uma posição central , $41 \%$ dos erros são cometidos na posição adjacente central $(F(2,20)=4,1$, $p=0,031$ ). Assim como os erros cometidos com as letras, os erros cometidos frente aos padrões visuais sem nome também sugerem que os chutes não são completamente ao acaso, embora nesta condição os erros sejam mais distribuídos nas diferentes posições.

Comparando os resultados obtidos com letras e padrões visuais, podemos constatar que a taxa de recordação dos padrões visuais é 26 pontos percentuais menor do que a recordação de letras $(F(1,10)=66,73, p<0,001)$. O efeito da similaridade é pequeno $(7 \%)(F(1,10)=14,38, p<0,001)$, e embora seja maior nas provas com padrões visuais $(10 \%)$ do que nas provas com letras $(3 \%)$ essa tendência não é significante $(F(1,10)=3,58, p=0,081)$.

Os resultados obtidos neste experimento replicam com perfeição os resultados obtidos por Walker e colaboradores (1993). A taxa de reconhecimento dos dois tipos de estímulos foi afetada pela similaridade visual, sugerindo que algum tipo de memória visual a curto prazo está envolvido tanto no armazenamento dos padrões visuais como no das letras. O efeito da similaridade visual sobre a taxa de reconhecimento de letras se restringe aos dois últimos estímulos apresentados, o que pode sugerir que no momento da apresentação do estímulo teste esses estímulos ainda mantinham traços de suas características visuais. Para os estímulos visuais sem nome o efeito da similaridade é maior para os estímulos da pré recência, sugerindo que esses estímulos ainda são armazenados em termos visuais quando o estímulo teste é apresentado.

Nossos resultados sugerem que as letras são codificadas, pelo menos durante um determinado tempo, em termos de seus atributos visuais. Durante esse tempo seriam sensíveis à similaridade visual, mas este fator deixaria de ser importante depois de os estímulos terem sido codificados em termos fonológicos, como pode ter acontecido aos primeiros estímulos apresentados.

Assim como nos estudos de D. E. Broadbent e M. H. P. Broadbent (1981) e de Walker e colaboradores (1993), com estímulos visuais sem nome, o efeito de recência sugere que a capacidade do sistema visual a curto prazo é maior do que a unidade. E, assim como nesses estudos, o efeito da similaridade sobre a taxa de acertos dos itens de prérecência pode ser considerada mais um argumento de que, ao contrário das letras, os primeiros padrões visuais ainda estariam armazenados na memória visual a curto prazo no momento da apresentação do estímulo teste.

A capacidade do sistema de memória visual a curto prazo se estenderia também a estímulos da pré-recência, de uma forma determinada, em parte, pela similaridade entre estímulos. Mas isso não esclarece porque apenas os últimos estímulos seriam mais bem lembrados, este dado não explica o efeito de recência.

\section{Discussão Geral}

Neste estudo investigamos a natureza das representações e dos recursos empregados pelo sistema de memória visuoespacial a curto prazo utilizando tarefas intervenientes de supressão articulatória verbal e de subtração aritmética. 
Nossos resultados mostram que a supressão articulatória verbal tem um efeito deletério sobre a recordação das letras, mas não afeta a recordação dos padrões visuais. Este resultado corrobora a idéia de que o armazenamento dos padrões visuais não depende, como o das letras, do laço articulatório, sugerindo que o armazenamento desses estímulos é realizado por um sistema de memória visual a curto prazo. $\mathrm{O}$ efeito deletério da similaridade sobre a recordação das posições das letras e dos padrões visuais sugere que esses estímulos são armazenados em termos de suas características visuais. A realização simultânea da tarefa aritmética afeta de forma significante as taxas de reconhecimento dos padrões visuais e das letras, e seu efeito é da mesma magnitude nos dois tipos de estímulos. Uma vez que a supressão verbal não afeta o desempenho dos padrões visuais, e que a tarefa aritmética tem o mesmo efeito nos padrões visuais e nas letras, podemos concluir que a tarefa aritmética deve utilizar os mesmos recursos que a codificação da posição espacial, ou seja, podemos concluir que a tarefa de subtração afeta o componente espacial do sistema de memória visuo-espacial a curto prazo.

É evidente que conclusões desse tipo dependem da natureza das tarefas investigadas. Uma tarefa aritmética pode tanto envolver o laço fonológico, no caso em que o participante soma números de um dígito (Noel, Désert, Aubrun \& Seron, 2001), como pode envolver o executivo central, no caso em que a soma envolve números com dois dígitos (Logie, Gilhooly \& Wynn, 1994). Técnicas de imageamento funcional também mostram que cálculo aritmético simples (Ex.: 16+6) envolve áreas normalmente associadas ao laço fonológico do modelo de memória operacional (Cowell, Egan, Code, Harasty \& Watson, 2000). Enquanto que a execução de cálculos complexos (Ex.: 32 x 24) é acompanhada de um aumento da ativação em áreas associadas ao processamento da informação visuoespacial (Zago \& cols., 2001). De acordo com o modelo de processamento numérico proposto por Dehaene (1992), tarefas de subtração, tal como a utilizada neste estudo, envolvem o uso de um código de magnitude analógico, que poderia estar envolvido no armazenamento do componente espacial da tarefa de localização que utilizamos.

Também é possível que a tarefa aritmética e a memória para localização sejam dependentes de recursos atencionais. Para Miyake, Friedman, Rettinger, Shah e Hegarty (2001) a relação entre a memória operacional visuo-espacial, o executivo central e habilidades espaciais é íntima. Seus estudos mostram que tarefas operacionais que envolvem o processamento e $\mathrm{o}$ armazenamento são intimamente ligadas ao funcionamento do executivo central e não são claramente distinguíveis deste (mas ver também Duff \& Logie, 1999). Embora a distinção entre esses componentes do sistema de memória operacional tenha ainda que ser aprofundada, a melhor evidência atualmente disponível para subdivisões adicionais dos recursos da memória operacional vem do domínio visuo-espacial, sugerindo que o processo espacial e o visual podem se basear em pools separados de recursos (veja Logie, 1995, para uma revisão recente sobre a divisibilidade da memória de trabalho em seus de componente espacial e visual). Por exemplo, os padrões de interferência obtidos com o paradigma da tarefa dupla mostraram que a manutenção da informação espacial na memória de trabalho pode ser prejudicada por uma tarefa espacial simultânea mas não por uma tarefa visual concorrente, enquanto que a manutenção da informação visual pode ser prejudicada por uma tarefa visual simultânea mas não por uma tarefa espacial simultânea (Ex.: Baddeley \& Lieberman, 1980, citados em Baddeley, 1986; Logie, 1986, 1995).

A supressão articulatória verbal, assim como a tarefa aritmética, não igualaram o desempenho obtido com as letras àquele obtido com os padrões visuais. A superioridade das letras, mesmo sob a supressão da articulação e com recursos atencionais drenados pela tarefa aritmética, sugere que o sistema de memória a curto prazo pode tratar de forma diferenciada os estímulos em função da experiência previa que tenha tido com eles. Como apontamos acima, também é possível que a tarefa de supressão empregada, embora impeça que os participantes utilizem a informação fonológica associada aos estímulos, permita que esses estímulos sejam nomeados. É provável que as letras sejam nomeadas automaticamente, e embora a recitação de seus nomes seja impedida pela supressão articulatória, essa informação pode contribuir para o melhor desempenho que esses estímulos tiveram. Nesse caso, é possível que o processamento semântico também estivesse envolvido na memória visuoespacial. Evidências neurofisiológicas também sugerem que letras teriam um processamento privilegiado em relação a outros estímulos visuais. Polk e colaboradores (2002), por exemplo, encontraram através de técnicas de imageamento cortical, uma área na região do giro fusiforme esquerdo que responde mais frente a consoantes do que frente a dígitos ou formas, demonstrando a especialização neural no cérebro humano para essa categoria de estímulos.

Os estudos de Phillips e Christie (1977a, 1977b) sugerem, com base na interação entre a memória visual e uma tarefa aritmética, que a retenção de estímulos visuais sem nome depende de recursos mais gerais do sistema, talvez de 
recursos provenientes um sistema equivalente ao executivo central. No entanto, os estudos realizados por Logie, Zucco e Baddeley (1990) mostram que o desempenho em uma tarefa de capacidade visual é muito afetado por uma tarefa concorrente visual, e pouco afetado por uma tarefa concorrente aritmética, enquanto que o desempenho numa tarefa de capacidade verbal é bastante afetado pela tarefa concorrente aritmética mas não pela tarefa concorrente visual. De acordo com esses autores esses resultados sugerem sistemas de armazenamento visual e verbal a curto prazo independentes. Nossos resultados corroboram a independência entre o processamento de estímulos verbais e de estímulos visuais.

O efeito de recência obtido neste estudo não pode ser atribuído a um sistema de armazenamento perceptivo/ sensorial tal como sugerido por Phillips (1983). Nossos dados foram obtidos em situações nas quais o intervalo entre os estímulos a serem recordados e o estímulo teste é maior do que aquele utilizado por Phillips; além disso, nossos estímulos foram apresentados em posições diferentes entre si e da posição na qual foi apresentado o estímulo teste. Esses aspectos permitem eliminar a interpretação do efeito de recência como decorrente de um decaimento da informação sensorial. De acordo com D. E. Broadbent e M. H. P. Broadbent (1981) os efeitos de recência e da similaridade entre estímulos obtidos em tarefas de memória visual a curto prazo indicam que esse sistema tem uma capacidade limitada, mas não descartam a possibilidade de que os itens prérecência possam ser mantidos por códigos não visuais. Nossos resultados eliminam essa possibilidade, uma vez que mostram que a informação fonológica não foi utilizada no armazenamento de padrões visuais sem nome, e provavelmente foi impedida pela supressão articulatória nas condições em que foram utilizadas as letras.

A interpretação do efeito de recência tem se mostrado uma tarefa difícil. Os estudos iniciais de Phillips e Christie (1977a, 1977b) identificaram o efeito de recência, restrito a um único estímulo, à capacidade do sistema de memória visual a curto prazo. D. E. Broadbent e M. H. P. Broadbent (1981) mostraram que a capacidade desse sistema de memória, estimada pelo efeito de recência, poderia se estender a dois ou três ítens. Walker e colaboradores (1993) apresentaram evidências de que a capacidade da memória visual a curto prazo se estende também aos itens de pré-recência. O mesmo pode ser dito a partir dos efeitos da similaridade que obtivemos neste estudo. No entanto, essa associação entre efeito de recência e capacidade de armazenamento deve ser considerada com cautela, uma vez que têm sido relatados casos de lesões cerebrais que afetam a capacidade de memória verbal, mas deixam intacto o efeito de recência, enquanto que lesões em outras áreas deixam intacta a capacidade de memória, mas eliminam o efeito de recência (Della Sala, Logie, Cubeli, Triveli \& Marchetti, 1998).

\section{Referências}

Baddeley, A. (1986). Working-memory. New York: Oxford University Press.

Baddeley, A. (2000). The episodic buffer: A new component of working memory? Trends in Cognitive Sciences, 4, 419-423.

Baddeley, A., Grant, W., Wight, E. \& Thompson, N. (1975). Imagery and visual working memory. Em S. Dornic (Org.), Attention and performance, $V$ (pp. 205-217). London: Academic Press.

Baddeley, A. \& Hitch, G. J. (1974). Working memory. Em G. Bower (Org.), The psychology of learning and motivation, VIII (pp. 47-90). New York: Academic Press.

Broadbent, D. E. \& Broadbent, M. H. P. (1981). Recency effects in visual memory. Quarterly Journal of Experimental Psychology, 33 A, 1-15.

Brooks, L. R. (1967). The suppression of visualization by reading. Quarterly Journal of Experimental Psychology, 19, 289-299.

Cowell, F., Egan, G. F., Code, C., Harasty, J. \& Watson, J. D. G. (2000). The functional neuroanatomy of simple calculation and number repetition: A parametric PET activation study. NeuroImage, 12, 565-573.

Dehaene, S. (1992). Varieties of numerical abilities. Cognition, 44, 1-24.

Della Sala, S., Logie, R. H., Cubelli, R., Trivelli, C. \& Marchetti, C. (1998). Dissociation between recency and span: Neuropsychological and experimental evidence. NeuroPsychology, 12, 533-545.

Duff, S. C. \& Logie, R. H. (1999). Storage and processing in visuo-spatial working memory. Scandinavian Journal of Psychology, 40, 251-259.

Galera, C. \& Munhemeze, A. Q. (1998). O processo de comparação na tarefa de classificação de caracteres: Evidências baseadas na similaridade e na posição do estímulo teste na lista memorizada. Arquivos Brasileiros de Psicologia, 50, 92-107.

Hitch, G. J., Halliday, M. S., Schaafstal, A. M. \& Schraagen, J. M. C. (1988). Visual working memory in young children. Memory and Cognition, 16, 120132.

Lee, K-M. \& Kang, S-Y. (2002). Arithmetic operation and working memory: Differential suppression in dual tasks. Cognition, 83, B63-B68.

Logie, R. H. (1986). Visuo-spatial processing in working memory. Quarterly Journal of Experimental Psychology, 38A, 229-247.

Logie, R. H. (1995). Visuo-spatial working memory. Hove, UK: LEA.

Logie, R. H., Gilhooly, K. J. \& Wynn, V. (1994). Counting on working memory in arithmetic problem solving. Memory \& Cognition, 22, 395-410.

Logie, R. H., Zucco, G. M. \& Baddeley, A. (1990). Interference with visual shortterm memory. Acta Psychologica, 75, 55-74.

Milner, D. A. \& Goodale, M. A. (1995). The visual brain in action. Oxford, UK: Oxford University Press.

Miyake, A., Friedman, N. P., Rettinger, D. A., Shah, P. \& Hegarty, M. (2001). How are visuospatial working memory, executive functioning, and spatial abilities related? A latent-variable analysis. Journal of Experimental Psychology: General, 130, 621-640.

Noel, M-P, Désert, M., Aubrun, A. \& Seron, X. (2001). Involvement of short-term memory in complex mental calculation. Memory \& Cognition, 29, 34-42.

Phillips, W. A. (1983). Short-term visual memory. Philosophical Transaction of the Royal Society, London, B302, 295-309.

Phillips, W. A. \& Christie, D. F. M. (1977a). Components of visual memory. Quarterly Journal of Experimental Psychology, 29, 117-133. 
Phillips, W. A. \& Christie, D. F. M. (1977b). Interference with visualization. Quarterly Journal of Experimental Psychology, 29, 637-650.

Polk, T. A., Stallcup, M., Aguirre, G. K., Alsop, D. C., D’esposito, M., Detre, J. A. \& Farah, M. J. (2002). Neural specialization for letter recognition. Journal of Cognitive Neuroscience, 14, 145-59.

Taylor, D. A. (1976). Holistic and analytic processes in the comparison of letters. Perception \& Psychophysics, 20, 187-190.

Ungerleider, L. G. \& Mishkin, M. (1982). Two cortical visual systems. Em D. J. Ingle, M. A. Goodale \& R. J. W. Mansfield (Orgs.), Analysis of visual behavior (pp. 549-586). Boston, MA: MIT Press.

Walker, P., Hitch, G. J. \& Duroe, S. (1993). The effect of visual similarity on shortterm memory for spatial location: Implications for the capacity of visual short-term memory. Acta Psychologica, 83, 203-224.
Zago, L., Pesenti, M., Mellet, E., Crivello, F., Mazoyer, B. \& Tzourio-Mazoyer, N. (2001). Neural correlates of simple and complex mental calculation. NeuroImage, 13, 314-327.

Sobre os autores

Cesar Galera é Professor Doutor do Departamento de Psicologia e Educação da Faculdade de Filosofia, Ciências e Letras de Ribeirão Preto, Universidade de São Paulo. Estuda memória a curto prazo e atenção visual.

Cláudia Correia Leite Fuhs é Mestre em Psicologia, Coordenadora do Curso de Psicologia da Universidade Paulista, campus de Ribeirão Preto. 